\title{
Transient anabolic effects accompany epidermal growth factor receptor signal activation in articular cartilage in vivo
}

\author{
John B Shepard ${ }^{1}$, Jae-Wook Jeong ${ }^{2}$, Nita J Maihle ${ }^{3}$, Sean O'Brien ${ }^{1}$ and Caroline N Dealy ${ }^{1,4^{*}}$
}

\begin{abstract}
Introduction: Signals from the epidermal growth factor receptor (EGFR) have typically been considered to provide catabolic activities in articular cartilage, and accordingly have been suggested to have a causal role in osteoarthritis progression. The aim of this study was to determine in vivo roles for endogenous EGFR signal activation in articular cartilage.

Methods: Transgenic mice with conditional, limb-targeted deletion of the endogenous intracellular EGFR inhibitor Mig-6 were generated using CreLoxP (Mig-6-flox; Prx1Cre) recombination. Histology, histochemical staining and immunohistochemistry were used to confirm activation of EGFR signaling in the articular cartilage and joints, and to analyze phenotypic consequences of Mig-6 loss on articular cartilage morphology, proliferation, expression of progenitor cell markers, presence of chondrocyte hypertrophy and degradation of articular cartilage matrix.

Results: The articular cartilage of Mig-6-conditional knockout (Mig-6-cko) mice was dramatically and significantly thicker than normal articular cartilage at 6 and 12 weeks of age. Mig-6-cko articular cartilage contained a population of chondrocytes in which EGFR signaling was activated, and which were three to four times more proliferative than normal Mig-6-flox articular chondrocytes. These cells expressed high levels of the master chondrogenic regulatory factor Sox9, as well as high levels of putative progenitor cell markers including superficial zone protein (SZP), growth and differentiation factor-5 (GDF-5) and Notch1. Expression levels were also high for activated $\beta$-catenin and the transforming growth factor beta (TGF- $\beta$ ) mediators phospho-Smad2/3 (pSmad2/3). Anabolic effects of EGFR activation in articular cartilage were followed by catabolic events, including matrix degradation, as determined by accumulation of aggrecan cleavage fragments, and onset of hypertrophy as determined by type $\times$ collagen expression. By 16 weeks of age, the articular cartilage of Mig-6-cko knees was no longer thickened and was degenerating.
\end{abstract}

Conclusions: These results demonstrate unexpected anabolic effects of EGFR signal activation in articular cartilage, and suggest the hypothesis that these effects may promote the expansion and/or activity of an endogenous EGFRresponsive cell population within the articular cartilage.

Keywords: Epidermal growth factor receptor, EGFR, Articular cartilage, Osteoarthritis, Progenitor cells, Chondroprogenitors, Cartilage repair, Mig-6

\footnotetext{
* Correspondence: dealy@nso2.uchc.edu

'Center for Regenerative Medicine and Skeletal Development, Department of Reconstructive Sciences, School of Dental Medicine, University of Connecticut Health Center, 263 Farmington Avenue, Farmington CT 06030, USA

Full list of author information is available at the end of the article
} 


\section{Introduction}

Because adult articular cartilage has limited intrinsic regenerative capacity, damage to the tissue due to trauma or long term use during aging is not naturally repaired, causing osteoarthritis [1-3]. Current clinical strategies for articular cartilage repair include cell-based approaches [4], such as Autologous Chondrocyte Implantation [5], in which donor or autologous adult chondrocytes are placed into focal articular cartilage defects; or microfracture [6], in which penetration of the subchondral bone beneath the defect allows influx of endogenous blood and bone marrow cells into the region. A disadvantage of both of these approaches is that the defects tend to be filled by fibrocartilage [7], which lacks the durability of hyaline cartilage. This is likely due to characteristics inherent in the repair cells, which include the poor proliferative capacity of adult or aged chondrocytes, and their tendency to de-differentiate [8]; and the cellular heterogeneity of bone marrow, which contains only a small percentage of progenitor cells capable of chondrogenic differentiation $[9,10]$. Accordingly, critical steps towards articular cartilage repair and osteoarthritis treatment will be to identify progenitor cells with the ability to form articular cartilage, and to understand the signals that control their proliferation and chondrogenic differentiation [11].

The superficial and/or middle zones of normal articular cartilage have been identified as regions enriched in cells which are highly proliferative and/or which express mesenchymal or progenitor cell markers [12-17]. In vitro differentiation assays have demonstrated the potential of these cells to differentiate into the chondrogenic lineage [12-18], and particularly, the permanent hyaline or articular cartilage lineage $[12,17,18]$. Thus, these populations have been suggested to represent a reserve capacity of the normal articular cartilage for homeostasis or regeneration [14-16].

It is apparent that endogenous progenitors present within the articular cartilage are inadequate for selfrepair, as they are observed in osteoarthritic cartilage $[14,15,17,19,20]$. It has been suggested that advanced age, which is typical of idiopathic osteoarthritis, may reduce the size and/or alter the activity of the progenitor cell pools $[19,21,22]$. Osteoarthritic cartilage exhibits quantitative and qualitative differences in the expression of progenitor markers compared to normal cartilage $[19,20]$, and cells expressing progenitor markers are markedly more abundant in fetal and juvenile articular cartilage than in articular cartilage from adult or elderly patients $[22,23]$. Thus, while progenitor cells offer exciting potential for articular cartilage repair and osteoarthritis treatment, there is a critical need to identify signals which promote expansion and/or activity of endogenous progenitor cell pools in the articular cartilage, and/or which stimulate chondrogenic potential by putative exogenous cartilage repair cells.

The epidermal growth factor receptor (EGFR) network is emerging as an important signaling family in cartilage development, homeostasis and disease [24-35]. EGFR signals typically suppress chondrogenic differentiation and/ or homeostasis. For example, in vitro studies show that EGFR signals suppress initial chondrogenic differentiation by limb mesenchymal cells $[27,28]$, and also suppress matrix synthesis and/or stimulate activity of matrix degradative enzymes by articular chondrocytes [29-32]. EGFR signals also promote the de-differentiation of articular chondrocytes in vitro towards fibrogenic cell types [33-35]. Together these observations demonstrate effects of EGFR signaling in suppression of articular cartilage homeostasis, and suggest that activation of EGFR signaling may be a causal factor in osteoarthritis. Consistent with this, EGFR signaling is increased in the articular cartilage of osteoarthritic patients [32], and in rats following experimental surgical osteoarthritis induction [36].

To better understand the function of EGFR signaling in articular cartilage in vivo, in this study we have developed a murine model in which activation of EGFR signaling is targeted to the developing and adult limbs, including the joints and articular cartilage, via limb mesoderm-targeted conditional loss of Mig-6, an endogenous intracellular inhibitor of EGFR signaling [37]. The articular cartilage of the knee joints of Mig-6-cko mice undergoes progressive osteoarthritis-like changes characterized by late-stage articular cartilage degradation, which is unexpectedly preceded by dramatic thickening of the articular cartilage. The articular cartilage of Mig-6-cko joints is thickest at six weeks of age, and articular cartilage thickening is preceded by pronounced EGFR signal activation, significantly enhanced proliferation, and expanded expression of the master chondrogenic regulatory factor Sox 9 and other markers of putative progenitor cells, which is observed within presumptive articular cartilage as early as postnatal Day 5. Our study demonstrates for the first time anabolic effects in articular cartilage occurring in association with EGFR signal activation, and suggests novel possibilities for future application for cartilage repair and osteoarthritis treatment.

\section{Materials and methods \\ Experimental animals}

To produce Mig-6 conditional loss targeted to the mesoderm of developing limb buds, the Prx1-Cre transgene, which drives recombination in early limb bud mesenchyme [38], was introduced into Mig-6-flox/flox mice [39]. Resultant Prx1-Cre;Mig-6-flox/+ male mice were mated with Mig-6-flox/flox female mice to obtain Mig-6 conditional knockout mice (Prx1-Cre; Mig-6-flox/flox). Mig-6flox/flox littermates were used as controls. Genotyping 
of the mice and embryos was by polymerase chain reaction (PCR) using DNA prepared from tail biopsies. All protocols for animal use were approved by the Animal Care Committee of the University of Connecticut Health Center, and were in accordance with NIH guidelines.

\section{Histology and staining}

Limbs were dissected from adult mice and immediately fixed in $4 \%$ paraformaldehyde and processed for paraffin embedding. Histological analysis was performed on 7$\mu \mathrm{m}$ sections. Safranin O staining of glycosaminoglycans was performed by staining sections with Weigert's Iron Hematoxylin and $0.02 \%$ aqueous Fast Green, followed by rinsing with $1 \%$ acetic acid and staining with $0.1 \%$ aqueous Safranin O.

\section{Immunohistochemistry}

Immunohistochemical staining was performed as previously described [40]. In brief, sections were de-paraffinized, rehydrated and incubated with $3 \%$ hydrogen peroxide in water for 15 minutes to quench endogenous peroxidases. After blocking with 10\% normal goat serum for rabbit antibodies or M.O.M blocking serum (Vector Laboratories, Burlingame, CA, USA) for mouse antibodies, the slides were incubated with primary antibodies in blocking buffer at $4^{\circ} \mathrm{C}$ overnight. Dilutions of primary antibodies were as follows: rabbit anti-Mig-6, (Sigma, St. Louis, MO, USA), 1:200; rabbit anti-pEGFR (Y1092), (Abcam, Cambridge, MA, USA), 1:250; rabbit anti-SZP (Novus Biologicals, Littleton, CO, USA), 1:100; rabbit anti-Ki67 (Abcam, Cambridge, MA, USA), 1:50; rabbit anti-Notch1 (Abcam), rabbit 1:100; rabbit anti-pSmad2/3 (R\&D Systems, Minneapolis, MN, USA), 1:100; anti-Sox9 (Abcam), 1:500; rabbit anti-Aggrecan Neoepitope (Pierce, Rockford, IL, USA), 1:100; mouse anti-collagen type $\times$ (Developmental Studies Hybridoma Bank, Iowa City, IA, USA), 1:100; mouse anti-Activated- $\beta$ Catenin (Millipore, Temecula, CA, USA), 1:100; goat anti-GDF-5 (R\&D Systems), 1:50. The slides were washed with TBS containing $0.1 \%$ Tween 20 and then incubated with 1:200 biotinylated goat anti-rabbit IgG (Vector Laboratories) or M.O.M. Biotinylated Anti-mouse Ig Reagent (Vector Laboratories). After washing, the slides were incubated with Vectastain Elite ABC Reagent (Vector Laboratories) and developed with DAB reagent (Vector Laboratories) followed by counterstaining with hematoxylin. For negative controls, the specific antibody was omitted; none showed a positive reaction.

\section{In situ hybridization}

The mouse Col10a1 probe (gift of B. Olsen, Harvard University, Boston, MA) was subjected to digoxigeninlabeling (Roche Molecular Biochemicals, Indianapolis, IN, USA) using the protocol described by the manufacturer. In situ hybridization was performed on serially sectioned tissue that had been fixed in $4 \%$ paraformaldehyde as previously described [40].

\section{Cell proliferation}

Proliferating cells were detected with rabbit anti-Ki67 (Abcam), 1:100. Cell proliferation was quantified using image analysis within Photoshop CS4 Extended (Adobe Systems Inc. San Jose, CA, USA in fixed areas of $20 \times$ digital photographs of adult Mig-6-flox/flox and Mig-6cko mice articular cartilage. Ki67-labeled cells within the fixed area were automatically selected by color range, and the number of labeled cells was determined manually using the image analysis 'Count Tool' within Photoshop. Four to six sections of tibial articular cartilage were examined from the knees of five different Mig-6-flox/flox and Mig-6-cko mice for each time point.

\section{Determination of articular cartilage thickness}

Articular cartilage thickness was determined by measuring the mean distance at its thickest point from the articular cartilage surface to the subchondral bone in Safranin-O stained sections. Images were taken at $40 \times$ from representative non-overlapping fields of knees from different Mig-6-flox/flox and Mig-6-cko mice $(n=$ 5 each at 12 weeks; $n=6$ each at 6 weeks).

\section{Statistical analysis}

Statistical analysis was performed using GraphPad Prism (GraphPad Software Inc., San Diego, USA, USA). For direct comparisons Mann-Whitney U tests were used.

\section{Results}

\section{Thickening of the articular cartilage of Mig-6-flox;} Prx1Cre knee joints

Histological analysis of the knee joints of Mig-6-flox; Prx1Cre (Mig-6-cko) mice revealed dramatic thickening of the articular cartilage (Figure 1). At 12 weeks, the articular cartilage of the tibial surfaces of control Mig-6flox mice was on average $162 \pm 15 \mu \mathrm{m}$ thick $(n=5)$, compared to the average thickness of the tibial articular cartilage of Mig-6-flox; Prx1Cre mice, which was $266 \pm$ $36 \mu \mathrm{m}$ thick $(n=5, P<0.01$, compare Figure $1 \mathrm{C}$ vs $\mathrm{D}$ and see graph in Figure $1 \mathrm{~F}$ ). The articular cartilage of the femoral surfaces of Mig-6-cko joints was also increased $(283 \pm 19 \mu \mathrm{m}$ thick for Mig-6-cko, $n=5$, compared to $132 \pm 16 \mu \mathrm{m}$ thick for control Mig-6-flox, $n=5, P<0.01$, data not shown). Histochemical staining revealed that Safranin-O positive staining was reduced in the superficial zone of the thickened Mig-6-cko articular cartilage (compare Figure 1C, D). The superficial zone of the articular cartilage of the Mig-6-cko joints was highly cellular and contained numerous rounded chondrocytes often appearing as doublets (Figure 1D). As shown in Figure $1 \mathrm{G}$ and $1 \mathrm{H}$, the articular cartilage of Mig-6-cko 

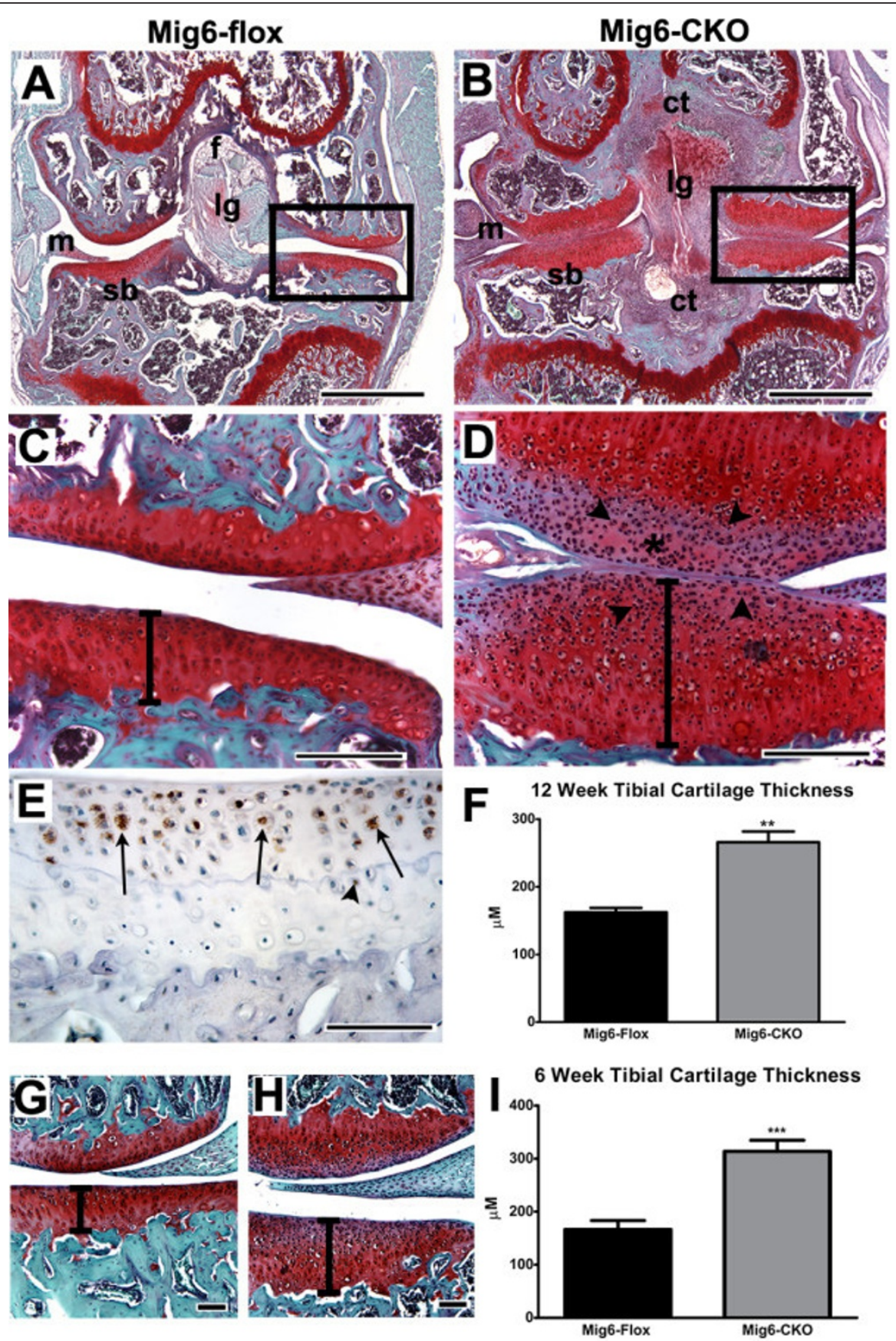

Figure 1 Articular cartilage thickness and Mig6 localization. (A-D) Sections of 12-week-old normal Mig-6-flox (A, C) and Mig-6-flox;PrxiCre conditional knockout (Mig-6-cko) knees (B, D) stained with Safranin-O to detect proteoglycan (red) and counterstained with Fast Green (tibia is at the bottom, femur is at the top). The articular cartilage of the tibial and femoral surfaces (boxed areas in A, B, shown at high magnification in $C, D)$ is dramatically thickened in the Mig-6-cko knee $(B, D)$. In addition, reduced Safranin-O staining is observed in the the superficial zone of the Mig-6-cko articular cartilage $(*$ in D), which is highly cellular and contains numerous rounded chondrocytes often appearing as doublets (arrowheads in D). Note also the thickened ligaments (lg) and menisci (m); abundant connective tissue (ct), and thin subchondral bone (sb) in Mig-6-cko knee joint (compare A to B). (E) Immunohistochemical detection of Mig-6 protein in 12-week-old normal Mig-6-flox tibial articular cartilage, showing Mig-6-positive chondrocytes (brown stain, arrows) mainly in the superficial zone. Some Mig-6-positive chondrocytes were also present in deeper zones (arrowhead) in the articular cartilage adjacent to the tidemark. (F) Measurement of the widths of the normal and Mig-6cko tibial articular cartilages (for example, see bars in C, D) shows that the Mig-6-cko articular cartilage is dramatically thicker than normal articular cartilage. The articular cartilage of Mig-6-cko mice was more than 1.5-fold thicker than normal articular cartilage at 12 weeks of age $(P<0.01)$. (G, H) Sections of six-week normal Mig-6-flox (G) and Mig-6-cko knees (H) stained with Safranin-O/Fast Green. The articular cartilage of Mig-6-cko joints is also dramatically thickened at six weeks of age (compare bars in G vs. H). (I) Measurement of the widths of the normal and Mig-6-cko tibial articular cartilages shows that the Mig-6-cko articular cartilage is nearly two-fold thicker at six weeks of age $(P<.001)$. Scale Bar $=$ $500 \mu \mathrm{m}(\mathrm{A}, \mathrm{B}) ; 200 \mu \mathrm{m}(\mathrm{C}, \mathrm{D}) ; 100 \mu \mathrm{m}(\mathrm{E}, \mathrm{G}, \mathrm{H})$. 
mice at 6 weeks was also dramatically thickened, and even thicker than at 12 weeks $(318 \mu \mathrm{M}+16.6 \mu \mathrm{m}$ thick for Mig-6-cko, $n=6$, compared to $170+20.9 \mu$ m thick for control Mig-6-flox, $n=6, P<0.001$, see graph in Figure 1I).

To confirm endogenous expression of Mig-6 protein in normal articular cartilage, immunohistochemical staining with a Mig- 6 antibody was performed, which demonstrated Mig-6 protein localization particularly in the superficial zone of the normal 12 week tibial (Figure 1E) and femoral (not shown) knee articular cartilages. Isolated Mig-6-positive chondrocytes were also located deep in the articular cartilage adjacent to the tidemark (Figure 1E) and in the subchondral bone (see Additional Figure 1A).

Mig-6-cko knee joints also contained thickened lateral and central ligaments which stained intensely with Safranin-O, abundant connective tissue, and enlarged menisci (Figure 1B). The subchondral bone present in the Mig-6-cko knee was thin and contained large bone marrow sinuses (Figure 1B).

\section{EGFR signaling in normal and Mig-6-flox;PrxCre articular cartilage}

Immunostaining with an antibody against the phosphorylated tyrosine residue 1092 of the EGFR kinase domain showed that EGFR signaling was occurring in normal articular cartilage, and increased in Mig-6-cko articular cartilage (Figure 2). In normal control Mig-6-flox knees, EGFR signaling was activated as early as postnatal Day 5 (the earliest day examined) in chondrocytes located in the distal region of the tibial epiphysis which will form the articular cartilage (Figure 2A). At six weeks of age EGFR signaling in normal tibial articular cartilage was limited to the superficial zone (Figure 2C). In the normal knee at 12 weeks of age, few superficial chondrocytes were EGFRpositive, but EGFR-positive chondrocytes were relatively abundant in the calcified zone adjacent to the chondroosseous junction, as well as in the subchondral bone itself (Figure 2E). In Mig-6-cko knee articular cartilage, EGFR signaling was dramatically enhanced in these regions compared to controls (Figure 2B, D, F). In addition, the domain of EGFR signal activation was expanded as early as postnatal Day 5 (compare bars in Figure 2A vs. Figure 2B), and EGFR-positive chondrocytes were abundant in the middle region of the Mig-6-cko articular cartilage at 6 and 12 weeks, a region which in controls contained few EGFR-positive chondrocytes (compare Figure $2 \mathrm{C}, \mathrm{E}$ to $\mathrm{D}, \mathrm{F}$ ). The patterns of EGFR activation were similar in femoral articular cartilage (not shown).




Articular chondrocyte proliferation in normal and Mig-6flox;Prx1Cre knee joints

Cell proliferation, as determined by immunostaining with a Ki67 antibody, was dramatically enhanced in the articular cartilage of Mig-6-cko knee joints compared to control Mig-6-flox knee joints (Figure 3). In control Mig- 6-flox tibia, only scattered proliferating cells were present in the presumptive articular cartilage at postnatal Day 5 (Figure 3A), and in the articular cartilage at 6 and 12 weeks of age (Figure 3C, E), and quantification of Ki67positive cells revealed that the level of proliferation remained constant over time (Figure 3G). In contrast, in

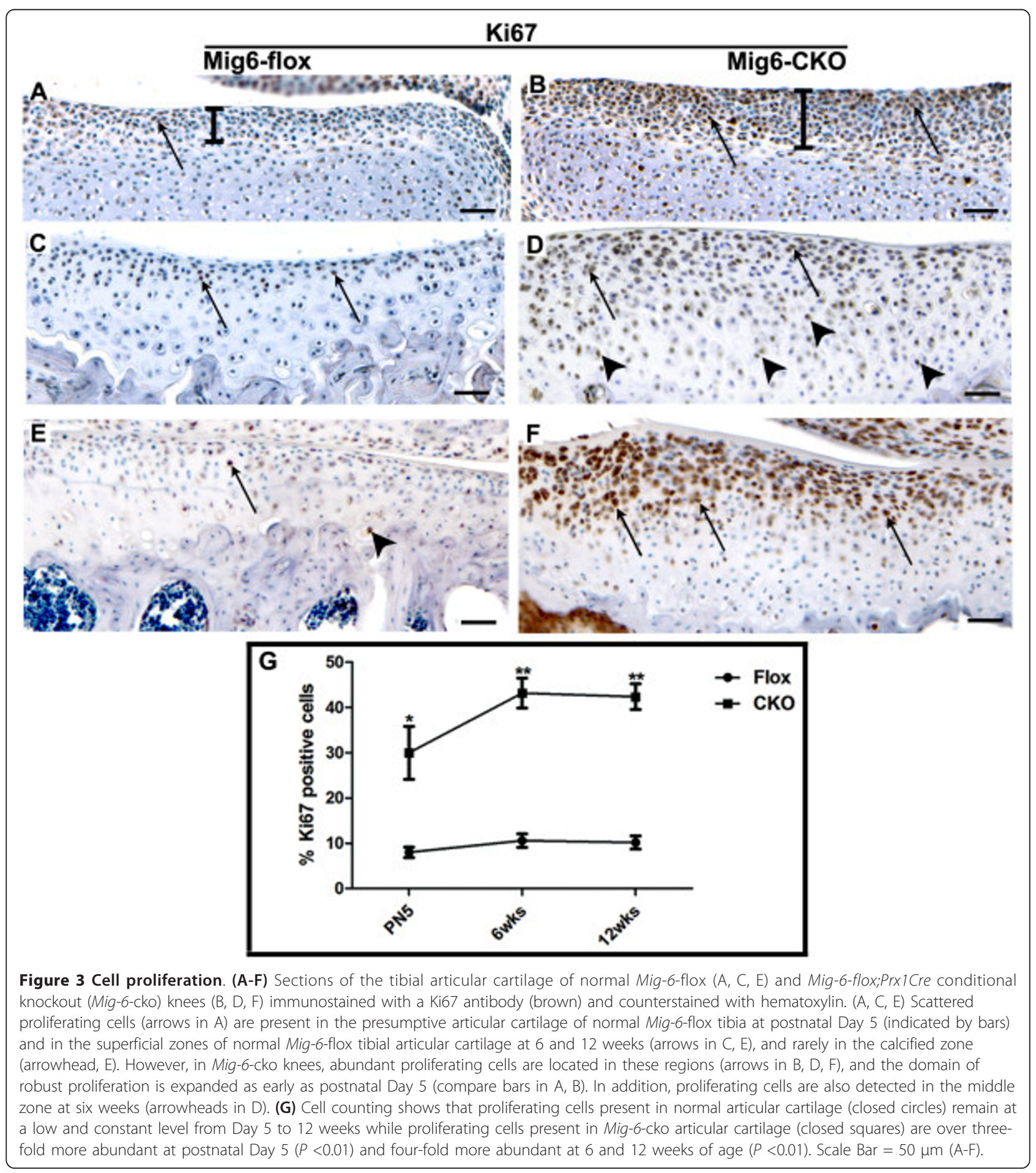


the Mig-6-cko knee, abundant proliferating cells were present in the presumptive articular cartilage at postnatal Day 5 , and in the superficial zones at 6 and 12 weeks, and the domain of robust proliferation is expanded as early as postnatal Day 5 (compare bars in Figure 3A vs. Figure $3 \mathrm{~B}$ ). In addition, proliferating cells were also present in deeper regions (Figure 3B, D, F). Cell counting revealed that the number of proliferating cells was about three times higher than controls at postnatal Day $5(P<$ $0.01, n=5$, Figure 3G), and four times higher than controls at 6 and 12 weeks of age $(P<0.01, n=5$, Figure 3G).

EGFR signal activation, increased proliferation, and tissue thickening were also observed in other regions of the Mig-6-deficient knee joint at six weeks of age (see Additional Figures 1 and 2). These regions include the central ligaments and especially the ligament/cartilage junctions (Additional file 1, Figure S1), as well as the menisci and synovium (see Additional file 2, Figure S2). Endogenous Mig-6 immunostaining was present in these tissues in normal six-week Mig-6-flox joints, but was not detected in any tissues including the articular cartilage, menisci, bone or ligament of six-week old Mig-6-cko joints (see Additional Figures 1 and 2).

\section{Expanded expression of progenitor cell markers in Mig-6-flox;Prx1Cre articular cartilage}

As shown by immunostaining, the relative abundance of cells expressing Sox9, superficial zone protein (SZP), growth and differentiation factor-5 (GDF-5), Notch1, activated $\beta$-catenin, and the transforming growth factor beta (TGF- $\beta$ ) mediators phospho-Smad2/3 (pSmad2/3), was markedly increased in Mig-6-cko articular cartilage compared to control articular cartilage (Figures 4 and 5). At 12 weeks of age, cells expressing these markers were present in the superficial zone of control Mig-6-flox tibial articular cartilage (Figure 4A, C, E and Figure 5A, C, E). However, in 12-week old Mig-6-cko tibial articular cartilage, cells expressing these markers were considerably more abundant and were present not only in the superficial but also in the middle zones (Figure 4B, D, F and Figure 5B, D, F). The distribution and relative abundance of these markers in Mig-6-cko femoral cartilage was also increased compared to control Mig-6-flox femoral articular cartilage (not shown). At six weeks of age, enhanced expression and expanded distribution of Sox9, Notch1, pSmad2/3 and SZP was also evident in Mig-6-cko articular cartilage (Figure 6H, J, L, N) compared to control Mig-6-flox articular cartilage (Figure 6G, I, K, M). Notably, an increased abundance and expanded distribution of cells expressing of Sox 9 , Notch 1 and $\mathrm{pSmad} 2 / 3$ protein relative to controls was also detected in the presumptive articular cartilage of Mig-6-cko at postnatal Day 5 , the earliest day examined (compare region shown by bars in Mig-6-flox (Figure 6A, C, E) vs Mig-6-cko
(Figure 6B, D, F). Measurement of the length of the bars indicates the region of expanded marker gene expression in the Mig-6-cko is approximately 25\% thicker than in normal Mig-6-flox controls.

\section{Matrix remodeling and chondrocyte hypertrophy in Mig-6-flox;Prx1Cre articular cartilage}

Little or no matrix turnover, as determined by immunostaining with an antibody to the aggrecan-cleavage fragment NITEGE, was detected in normal Mig-6-flox tibial articular cartilage at 6 and 12 weeks of age (Figure 7A-D). Safranin-O staining in normal Mig-6-flox tibial articular cartilage was also uniform at 6 and 12 weeks. In contrast, Safranin-O staining was reduced in the superficial zone of Mig-6-cko tibial articular cartilage, and this region contained immunoreactive NITEGE cleavage fragments (Figure 7E-H). The intensity of NITEGE immunostaining at 6 weeks was low, and became considerably increased by 12 weeks in Mig-6-cko articular cartilage (compare Figure 7G to H). Little or no NITEGE-positive immunostaining was observed in either normal or Mig-6-deficient presumptive articular cartilage at postnatal Day 5 (not shown). Few hypertrophic chondrocytes, detected through immunostaining for type $\times$ collagen and/or by in situ hybridization with a type $\times$ collagen probe, were observed in the articular cartilage of either normal Mig6-flox or Mig-6-cko knees at six weeks (Figure 7I-L). However, at 12 weeks, while few hypertrophic chondrocytes were detected in normal Mig-6-flox knees, several hypertrophic chondrocytes were observed in the articular cartilage of Mig-6-cko knees (compare Figure 7M, N to Figure $7 \mathrm{O}, \mathrm{P})$.

\section{Late stage degradation in Mig-6-flox;Prx1Cre articular cartilage}

At 16 weeks of age, Mig-6-cko articular cartilage was no longer overtly thickened and degradation of the articular cartilage along with gross joint abnormality was present (Figure 8). The tibial articular cartilage of Mig-6-cko knee joints at 16 weeks was comparable in thickness to normal articular cartilage at that age (Figure 8), but was reduced in thickness compared to Mig-6-cko articular cartilage at 12 and 6 weeks of age (compare Figure 8D to Figures 1D, H). In addition, the tibial articular cartilage was discontinuous, with loss of integrity both at the surface and at the chondro-osseous junction (Figure 8D). In some regions of the joint, it was not possible to detect a clear separation between the tibial articular cartilage surface and the meniscal fibrous tissue that filled the inter-articular space (Figure 8D). The knee joints of 16-week-old Mig-6-cko mice also contained fused and highly chondrified central ligaments; thickened and fibrogenic menisci; reduced subchondral bone area; and prominent central and lateral osteophytes (Figure 8B). 




\section{Discussion}

As EGFR signals have typically been reported to have negative roles in cartilage differentiation and homeostasis [27-36], our observation that in vivo activation of EGFR signaling leads to transient thickening of the articular cartilage is unexpected, and suggests potential novel anabolic functions for EGFR signals in cartilage tissue. The articular cartilage thickening that accompanies EGFR activation is also accompanied by increased proliferation of cells within the articular cartilage. EGFR signals have well-established mitogenic roles for many progenitor cell types, including mesenchymal progenitors $[41,42]$, and our previous studies have shown that EGFR signals stimulate in vitro and in vivo proliferation by embryonic limb mesenchymal cells $[27,43]$, and are also required for in vivo proliferation of immature chondrocytes in developing limb skeletal elements [44].
As proliferation is a requirement for chondrogenic differentiation by progenitor cells [45], our observation that activation of EGFR signaling stimulates proliferation in the articular cartilage, and especially in the superficial layers, which are enriched in progenitor cells [13-17], is consistent with an important role for endogenous EGFR signals in providing these pro-proliferative cues.

Progenitor cell populations present in the articular cartilage have been identified based on their expression of cell surface mesenchymal progenitor markers [14,15]; and/or expression of Notch1, Sox9, superficial zone protein (SZP) (aka prg4/lubricin), and growth and differentiation factor5 (GDF-5) [12,13,16-18,46], which have been implicated in cartilage or articular cartilage lineage differentiation, and/ or maintenance of chondrogenic potential $[16,18,46-48]$. Although definitive markers for articular cartilage progenitors are lacking [19], our observation that Mig-6-deficient 


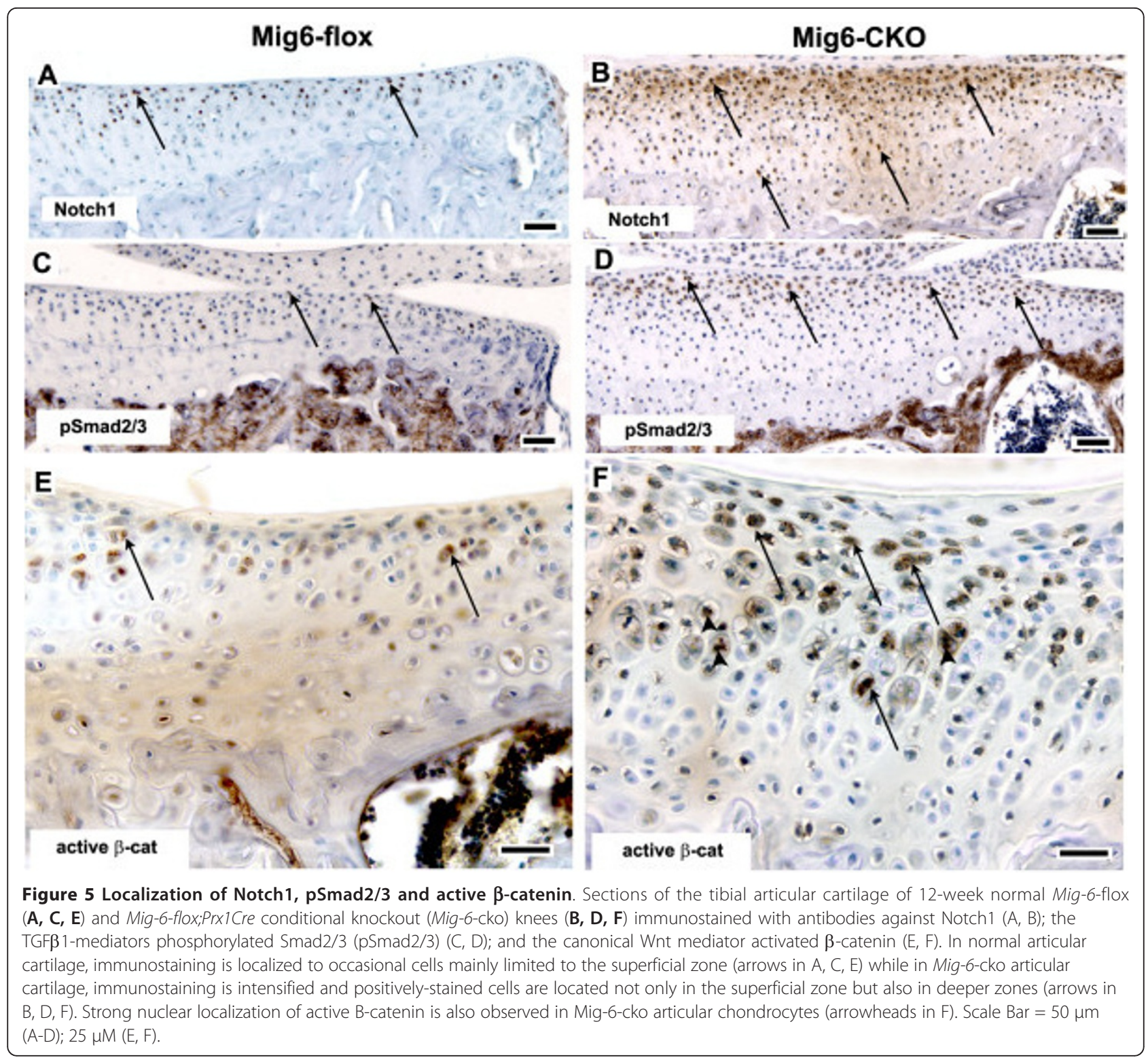

articular cartilage contains a population of cells which are highly proliferative and which express Notch1, Sox9, SZP and GDF- 5 suggests the existence of an endogenous EGFR-responsive progenitor cell pool in articular cartilage. These putative progenitor cells appear to have the potential to differentiate and contribute to the thickened Mig-6cko articular cartilage, as the chondroprogenitor marker and master chondrogenic regulator, Sox9, is up-regulated by the cells as they transition from the superficial zone into deeper regions of the articular cartilage.

The EGFR-responsive putative progenitor cells we observe in Mig-6-deficient articular cartilage also express increased levels of the TGF- $\beta$ mediators $\mathrm{pSmad} 2 / 3$, as well as high levels of nuclear-localized activated $\beta$-catenin, suggesting TGF- $\beta$ and canonical Wnt signaling pathways are stimulated in these cells. This is consistent with the proposed roles for these pathways as key regulators of articular cartilage progenitor cell and/or articular chondrocyte phenotypes $[18,49]$. For example, in vitro, articular cartilage superficial zone cells have been shown to proliferate and express progenitor or superficial zone markers in response to TGF- $\beta 1$ [50] and to transient activation of canonical Wnt signaling [18]; and in vivo, transient activation of $\beta$-catenin signaling, which like the EGFR has typically been associated with osteoarthritis [51] also causes articular cartilage thickening in postnatal mice [52]. Intriguingly, synergistic interactions occur among the TGF- $\beta$, Wnt and EGFR network in other systems [53-57]. The colocalization of $\mathrm{pSmad} 2 / 3$ and activated $\beta$-catenin by cells in the Mig-6-cko articular cartilage in which EGFR 


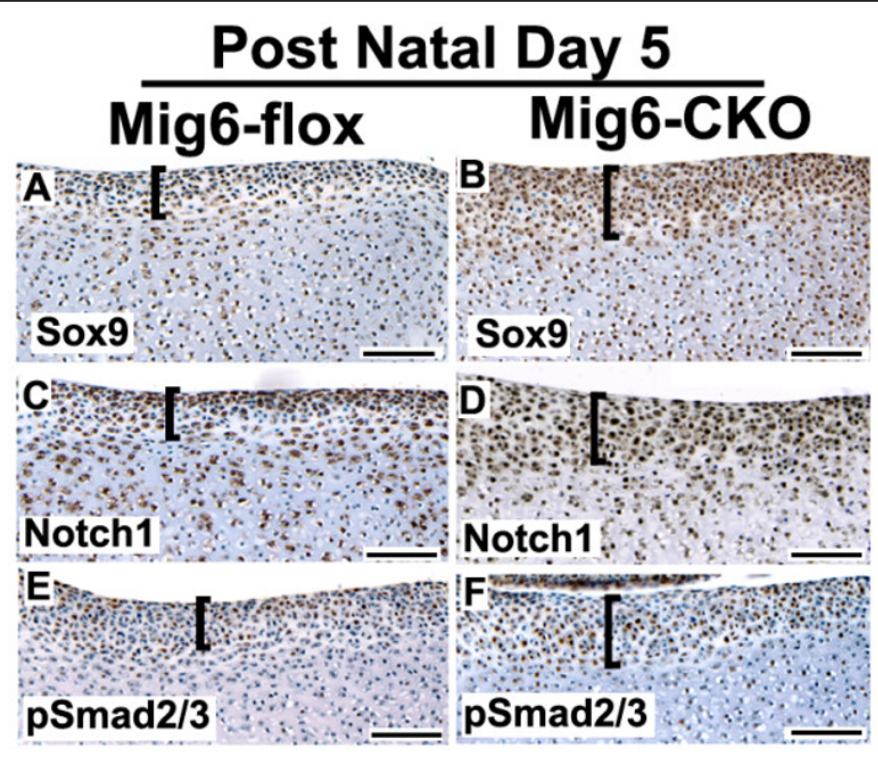

\section{Weeks}

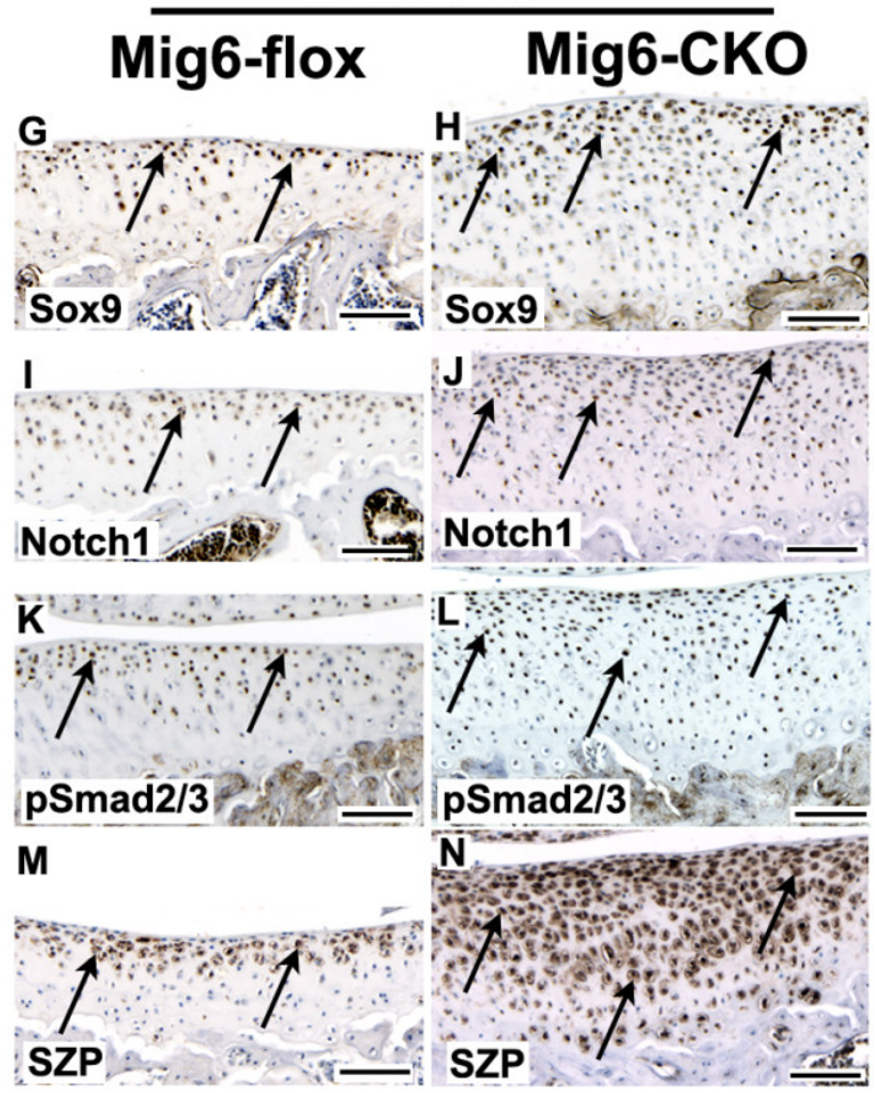

Figure 6 Time-dependent expansion of marker gene expression. (A-F) Sections of the distal tibial epiphysis of normal Mig-6-flox (A, C, E) and Mig-6-flox;Prx1Cre conditional knockout (Mig-6-cko) (B, D, F) at postnatal Day 5, immunostained with antibodies against Sox9 (A, B); Notch1 (C, D); or the TGF $\beta 1$-mediators phosphorylated Smad2/3 (pSmad2/3) (E, F). Immunostaining in the presumptive articular cartilage of the normal Mig-6-flox tibia is limited to a distal portion of the epiphyses (indicated by bars in A, C, E). However, immunostaining is intensified in the presumptive articular cartilage of the Mig-6-cko tibia, and the region of immunostaining is expanded (compare bars in B, D, F to A, C, E). (G-N) Sections of six-week old tibial articular cartilage of normal Mig-6-flox $(\mathbf{G}, \mathbf{l}, \mathbf{K}, \mathbf{M})$ and Mig-6-cko $(\mathbf{H}, \mathbf{J}, \mathbf{L}, \mathbf{N})$ joints immunostained with antibodies against Sox9 $(G, H)$; Notch1 (I, J); pSmad2/3 (K, L) or superficial zone protein (SZP, M, N). Enhanced marker expression by cells in the superficial and middle zones is evident in the six-week-old Mig-6-cko articular cartilage compared to control Mig-6-flox articular cartilage (arrows). Scale bar $=100 \mathrm{~nm}$. 


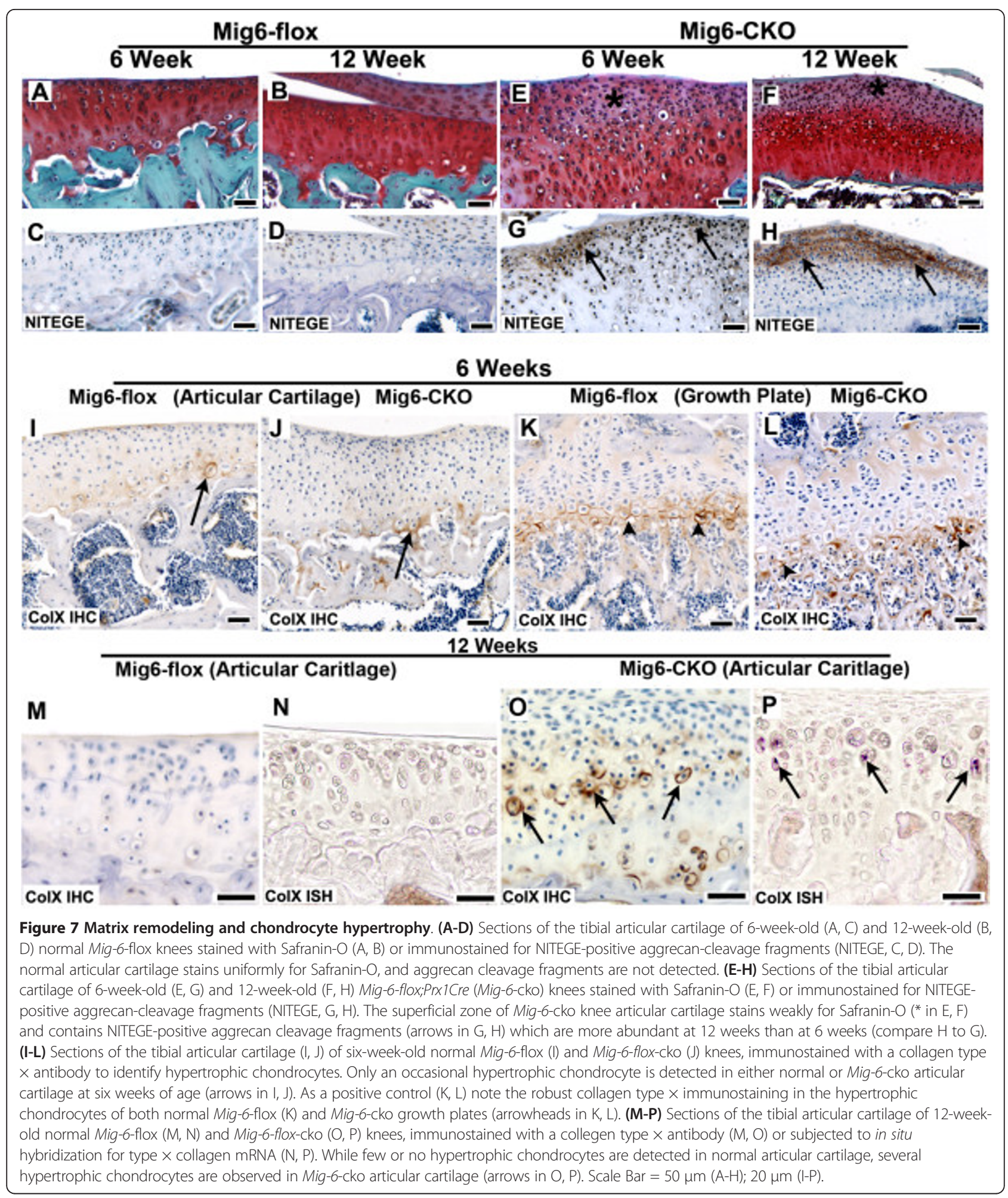

signaling is also activated suggests that expansion or activation of putative progenitor cells within the articular cartilage may involve interactions between the EGFR network and the TGF- $\beta$ and canonical Wnt networks.
Mig-6 is an intracellular inhibitor of EGFR signaling [37] which binds to the intracellular kinase domain of the EGFR [58]. One of the roles of Mig-6 is as a tumor suppressor gene [59], and in accordance with the well-established 




involvement of EGFR signaling in oncogenic progression [60], mice with global Mig-6 loss experience widespread and precocious tumor development [61]. Thus, it has been suggested that Mig-6 mediated inhibition of EGFR signals has evolved to control potentially inappropriate proliferative responses following cellular injury or stress [59]. Notably, Mig-6 is up-regulated in response to mechanical stress [62], and mice with global Mig-6 loss have previously been reported to develop early-onset degenerative joint disease in their load-bearing joints [26]. The reported knee joint phenotype of mice with global Mig-6 loss is similar to what we have observed in Mig-6-cko mice, including the presence of fibrous tissue and osteophytes within the joint, and loss of proteoglycan staining and eventual degradation of the articular cartilage [26]. The present study extends these findings by revealing previously unsuspected anabolic effects accompanying Mig- 6 loss and EGFR signal activation in articular cartilage, and by suggesting the presence of a putative progenitor cell population in the articular cartilage that is expanded in response to Mig-6 loss. Our observations suggest that release of Mig-6-mediated inhibition of EGFR signaling in Mig-6-cko articular cartilage activates EGFR-mediated anabolic responses by stimulating the proliferation and expansion of what we suggest are progenitor populations within the articular cartilage.

It is important to point out that as Mig-6 functions are downstream of ligand activation of the EGFR, Mig-6 loss does not result in constitutive or ligand-independent EGFR activation, but rather represents de-repression of endogenous ligand-bound receptor signals $[37,58]$. 
The endogenous expression of Mig-6 in chondrocytes, mainly in the superficial zone of normal adult murine articular cartilage, closely matches that of endogenous EGFR signaling, and is consistent with activation of EGFR signaling in this region following Mig-6 loss. As few Mig6-positive chondrocytes were detected outside the superficial zone in normal articular cartilage, it is possible that the enhanced EGFR signal activation we observed in deeper regions of the Mig-6-cko articular cartilage is due to release of Mig-6-inhibition by cells expressing Mig-6 near or below the limit of immunohistochemical detection, and/or is the result of proliferative expansion of the superficial zone cells which originally expressed it. The three- to four-fold increase in proliferative rate by superficial and middle zone cells in Mig-6-cko articular cartilage is consistent with this latter possibility.

The nature of the endogenous ligand-receptor interactions mediating the EGFR responses we have observed in Mig6-deficient articular cartilage is unknown. For example, while the EGFR ligands transforming growth factor alpha (TGF- $\alpha$ ), and EGF are expressed by articular chondrocytes $[32,63]$, studies typically implicate their functions in catabolic effects of EGFR signaling associated with osteoarthritic damage [29-32], rather than the anabolic effects we have observed here. As distinct EGFR signal outputs may be generated by differential ligand activation [64], it is possible that anabolic EGFR activities could be mediated by ligands other than EGF or TGF- $\alpha$; alternately, anabolic vs. catabolic EGFR activities in articular cartilage could be related to differences in the timing or level of EGFR activation achieved in in vitro studies vs. our in vivo studies. Choice of heterodimerization partner within the EGFR network can also influence signal output [65], indicating additional involvement from other EGFR-related receptors could also occur. In addition, Mig- 6 can directly bind to and inhibit signal transduction by the EGFR-related receptor, ErbB2 [66]. Some EGFR-independent effects of Mig-6 have been reported including direct inhibition of ERK [67] and hepatocyte growth factor (HGF)/Met signaling [68]; however, HGF is not a potent regulator of anabolic or catabolic gene expression in articular chondrocytes [69]. Our observation that EGFR signaling is dramatically increased in Mig-6-cko articular cartilage in the same regions where we observe major phenotypic effects is consistent with a potentially primary role for the EGFR in mediating most, if not all, of the articular cartilage responses we have observed.

The catabolic effects of EGFR signaling in mature articular chondrocytes in vitro include de-differentiation towards fibrogenic cell types [33-35]. Conceivably then, a possible explanation for the thickening of the Mig-6cko articular cartilage could be that EGFR signal activation results in de-differentiation and proliferation of mature articular chondrocytes. However, we favor a view that articular cartilage thickening in Mig-6-cko mice results from stimulation of an endogenous progenitor cell response, rather than a de-differentiative response by mature cells. In support of this view are our observations that enhanced EGFR signal activation, increased proliferation, and expanded expression of progenitor cell markers, occur as early as postnatal Day 5, at which stage the articular cartilage is not morphologically distinct and is considered immature. Indeed, at postnatal Day 5, the presumptive articular cartilage consists only of a superficial layer, and the middle and deeper zones are not yet formed [70]. Thus, we believe it is very likely that the time-dependent thickening of Mig-6cko articular cartilage is due to expansion and proliferation of an endogenous EGFR-responsive progenitor population present in the articular cartilage and especially the superficial zone. If true, this would suggest previously unsuspected activities for EGFR signaling in promoting progenitor cell responses in articular cartilage, which could have important potential utility for cartilage repair and regenerative medicine.

Ultimately, catabolic effects of sustained EGFR activation in Mig-6-cko articular cartilage predominate over anabolic ones, eventually causing thinning, loss of integrity and degradation of the articular cartilage. One possible explanation for these degenerative changes is that the immature cartilage matrix present in the articular cartilage surface layer may be insufficient to withstand cumulative loading to the joints. It is also possible that the increased matrix enzyme activity in Mig-6-cko articular cartilage we have observed eventually outpaces deposition of new matrix by the EGFR-responsive progenitor-derived cells. Indeed, sustained matrix degradation is considered to be a turning point in osteoarthritic progression leading to irreversible cartilage damage [71]. Consistent with this possibility, high-level activation of matrix enzymes occurs in the Mig6-cko articular cartilage at 12 weeks, shortly before overt degradation and thinning of the articular cartilage. Activation of chondrocyte hypertrophy in the articular cartilage is also considered to be part of the disease pathology leading to articular cartilage degeneration [72]. Consistent with this, hypertrophic chondrocytes are observed in Mig6-cko articular cartilage, but not in normal Mig-6-flox articular cartilage, at 12 weeks of age, shortly before overt degradation of the articular cartilage occurs. These observations suggest the hypothesis that EGFR signal activation has dual effects in articular cartilage, including an initial anabolic stimulation mediated by expansion of progenitor cells, which is followed by inappropriate activation of matrix remodeling and chondrocyte hypertrophy, leading to articular cartilage degradation and overt joint disease. It is important to point out that at six weeks of age, which is when the Mig-6-cko articular cartilage is thickest, and 
proliferation is greatest, hypertrophic chondrocytes are not yet detected. This suggests that anabolic effects of EGFR signal activation precede catabolic ones, and are not necessarily coincident. Accordingly, an intriguing consideration is the possibility that transient activation of EGFR signaling might result in stimulation of anabolic activities, perhaps without catabolic ones, which could suggest novel future utility for EGFR signal activation in strategies for articular cartilage repair and osteoarthritis treatment. Additional studies are needed to clarify whether anabolic effects resulting from EGFR activation can result in formation of functional articular cartilage tissue.

\section{Conclusions}

Our study provides in vivo evidence for the involvement of EGFR signal activation in regulating potentially distinct anabolic and catabolic activities in articular cartilage, and demonstrates that the intracellular inhibitor Mig-6 normally functions to limit these activities. Release of Mig-6-mediated inhibition of EGFR signals leads to an initial, transient, thickening of the articular cartilage accompanied by proliferation and expansion of an EGFR-responsive cell population, which expresses high levels of the master chondrogenic regulatory factor Sox9, as well as high levels of other putative progenitor markers. In the presence of sustained EGFR activation, these anabolic effects are followed subsequently by accelerated catabolic effects (matrix degradation and hypertrophy) which may contribute to the eventual loss of the articular cartilage in this model.

\section{Additional material}

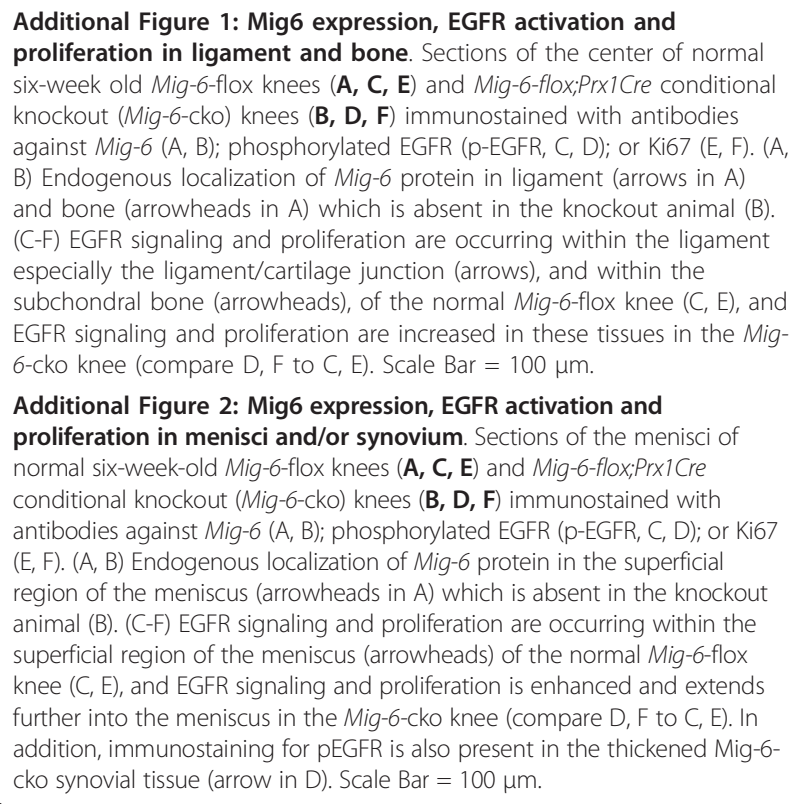

\section{Abbreviations}

EGF: epidermal growth factor; EGFR: epidermal growth factor receptor; GDF5: growth and differentiation-5; HGF: hepatocyte growth factor; HB-EGF: heparin-binding epidermal growth factor; Mig-6-cko: Mig-6 conditional knock out; pSmad2/3: phospho-Smad2/3; SZP: superficial zone protein; TGF-a: transforming growth factor alpha; TGF- $\beta$ : transforming growth factor beta

\section{Competing interests}

CD is a principal in a University of Connecticut faculty start-up called Chondrogenics, Inc. The goal of this company is to develop the potential of human embryonic and induced pluripotent stem cells for osteoarthritis treatment. Chondrogenics is funding a subcontract to CD's lab at the University of Connecticut to perform studies related to the company's goal. $C D$ does not receive any direct salary, fee or reimbursement from the company. Chondrogenics has not funded the research described in this manuscript. However, Chondrogenics could benefit in the future from the conceptual information described in this manuscript, and is contributing to the cost for publication of this manuscript. No other authors declare competing interests.

\section{Authors' contributions}

JS participated in acquisition, analysis and interpretation of data, and in manuscript preparation. JJ and NJM participated in data interpretation and manuscript preparation. SO participated in data acquisition and analysis. CND participated in experimental concept and design, data analysis and interpretation, and manuscript preparation. All authors read and approved the final manuscript

\section{Acknowledgements}

$\mathrm{NIH} / \mathrm{NICHD}$ PO1 HD-022610 (to CND). JS is supported by NIH/NIDCR training grant T90DE021989.

\section{Authors' details}

${ }^{1}$ Center for Regenerative Medicine and Skeletal Development, Department of Reconstructive Sciences, School of Dental Medicine, University of Connecticut Health Center, 263 Farmington Avenue, Farmington CT 06030, USA.

${ }^{2}$ Departments of Obstetrics, Gynecology and Reproductive Biology, Michigan State University, 333 Bostwick Avenue NE, Grand Rapids, MI 49503, USA.

${ }^{3}$ Departments of Obstetrics and Gynecology, Pathobiology, and Pharmacology, Yale University, 310 Cedar Street, New Haven, CT 06520, USA. ${ }^{4}$ Department of Orthopaedic Surgery, School of Medicine, University of Connecticut Health Center, 263 Farmington Avenue, Farmington, CT 06030, USA.

Received: 1 October 2012 Revised: 17 February 2013

Accepted: 25 May 2013 Published: 25 May 2013

\section{References}

1. Busija L, Bridgett L, Williams SR, Osborne RH, Buchbinder R, March L, Fransen M: Osteoarthritis. Best Pract Clin Rheumatol 2010, 24:757-768.

2. Lotz MK, Fraus VB: New developments in osteoarthritis. Arthritis Res Ther 2010, 12:211-220, Erratum in Arthritis Res Ther 2010, 12:408. Kraus, Virginia B [added].

3. Sun HB: Mechanical loading, cartilage degradation, and arthritis. Ann NY Acad Sci 2010, 1211:37-50.

4. Fisher MC, Ferrari D, Yincui L, Shepard JB, Patterson SE, Anderson N, Dealy CN: The potential of human embryonic stem cells for articular cartilage repair and osteoarthritis treatment. Rheumatol Curr Res 2012, S3.

5. Brittberg $M$, Lindahl A, Nilsson A, Ohlsson C, Isaksson O, Peterson L: Treatment of deep cartilage defects in the knee with autologous chondrocyte transplantation. N Engl I Med 1994, 331:889-895.

6. Gomoll AH: Microfracture and augments. J Knee Surg 2012, 25:9-15

7. Benthien JP, Schwaninger M, Behrens P: We do not have evidence based methods for the treatment of cartilage defects in the knee. Knee Surg Sports Traumatol Arthrosc 2011, 19:543-552.

8. Aigner T, Söder S, Gebhard PM, McAlinden A, Haag J: Mechanisms of disease: role of chondrocytes in the pathogenesis of osteoarthritisstructure, chaos and senescence. Nat Clin Pract Rheumatol 2007, 3:391-399. 
9. Estes BT, Diekman BO, Gimble JM, Guilak F: Isolation of adipose derived stem cells and their induction to a chondrogenic phenotype. Nat Protoc 2010, 5:1294-1311.

10. LaPrade RF, Bursch LS, Olson EJ, Havlas V, Carlson CS: Histologic and immunohistochemical characteristics of failed articular cartilage resurfacing procedures for osteochondritis of the knee: a case series. Am J Sports Med 2008, 36:360-368.

11. Dealy $\mathrm{CN}$ : Chondrogenic progenitors for cartilage repair and osteoarthritis treatment. Rheumatol Curr Res 2012, S3.

12. McCarthy HE, Bara JJ, Brakspear K, Singhrao SK, Archer CW: The comparison of equine articular cartilage progenitor cells and bone marrow-derived stromal cells as potential cell sources for cartilage repair in the horse. Vet J 2012, 192:345-351.

13. Hattori S, Oxford C, Reddi AH: Identification of superficial zone articular chondrocyte stem/progenitor cells. Biochem Biophys Res Comm 2007, 358:99-103.

14. Pretzel D, Linss S, Rochler S, Endres M, Kaps C, Alsalameh S, Kinne RW: Relative percentage and zonal distribution of mesenchymal progenitor cells in human osteoarthritic and normal cartilage. Arthritis Res Ther 2011, 13:R64.

15. Fickert S, Fiedler J, Brenner RE: Identification of subpopulations with characteristics of mesenchymal progenitor cells from human osteoarthritic cartilage using triple staining for cell surface markers. Arthritis Res Ther 2004, 6:R422-R432.

16. Dowthwaithe GP, Bishop JC, Redman SN, Khan IM, Rooney P, Evans DJR, Haughton L, Bayram Z, Boyer S, Thomson B, Wolfe MS, Archer CW: The surface of articular cartilage contains a progenitor population. J Cell Sci 2004, 117:889-897.

17. Williams R, Khan IM, Richardson K, Nelson L, McCarthy HE, Analbelsi T, Singhrao SK, Dowthwithe GP, Jones RE, Baird DM, Lewis H, Roberts S, Shaw HM, Dudhia J, Fariclough J, Briggs T, Archer CW: Identification and clonal characterization of a progenitor cell sub-population in normal human articular cartilage. PLOS One 2010, 5:e513246.

18. Yasuhara R, Ohta Y, Yuasa T, Kondo N, Hoang T, Addya S, Fortina P, Pacifici $M$, Iwamoto $M$, Enomoto-Iwamoto $M$ : Role of $\beta$-catenin signaling in phenotypic expression and proliferation of articular cartilage superficial zone cells. Lab Invest 2011, 91:1739-1752.

19. Grogan SP, Miyaki S, Asahara H, D'Lima DD, Lotz MK: Mesenchymal progenitor cell markers in human articular cartilage: normal distribution and changes in osteoarthritis. Arthritis Res Ther 2009, 11:R85.

20. Koelling S, Kruegel J, Immer M, Path JR, Sadowski B, Miro X, Miosge N: Migratory chondrogenic progenitor cells from repair tissue during the later stages of human osteoarthritis. Cell Stem Cell 2009, 4:324-335.

21. Martin JA, Buckwalter JA: The role of chondrocyte senescence in the pathogenesis of osteoarthritis and in limiting cartilage repair. J Bone Joint Surg Am 2003, 85:106-110.

22. Chang HX, Yang L, Li Z, Chen G, Dai G: Age-related biological characterization of mesenchymal progenitor cells in human articular cartilage. Orthopedics 2011, 34:e382-8.

23. Adkisson HD, Martin JA, Amendola RL, Milliman C, Mauch KA, Katwal AB, Syedin M, Amendola A, Streeter PR, Buckwalter JA: The potential of human allogenic juvenile chondrocytes for restoration of articular cartilage. Am J Sports Med 2010, 38:1324-1333.

24. Zhang $X$, Siclari VA, Lan S, Zhu J, Koyama E, Dupuis HL, Enomoto-Iwamoto M, Beier $F$, Qin $L$ : The critical role of the epidermal growth factor receptor in endochondral ossification. J Bone Miner Res 2011, 11:2622-2633.

25. Schneider MR, Sibilia M, Erben RG: The EGFR network in bone biology and pathology. Trends Endocrinol Metab 2009, 20:517-524.

26. Zhang YW, Su Y, Lanning N, Swiatek PJ, Bronson RT, Sigler R, Martin RW, Vande Woude GF: Targeted disruption of Mig- 6 in the mouse genome leads to early onset degenerative joint disease. Proc Natl Acad Sci USA 2005, 102:11740-11745.

27. Dealy CN, Scranton V, Cheng H-C: Roles of transforming growth factor-a and epidermal growth factor in chick limb development. Dev Biol 1998, 202:43-55

28. Yoon YM, Oh CD, Kim DY, Lee YS, Park JW, Hub TL, Kang SS, Chun JS: Epidermal growth factor negatively regulates chondrogenesis of mesenchymal cells by modulating the protein kinase c-alpha, Erk1 and p38MAPK signaling pathways. J Biol Chem 2000, 275:12353-12359.

29. Verschure PJ, Joosten LA, van der Kraan PM, Van den Berg WB: Responsiveness of articular cartilage from normal and inflamed mouse knee joints to various growth factors. Ann Rheum Dis 1994, 53:455-460.
30. Klooster A, Bernier SM: Tumor necrosis factor alpha and epiderma growth factor act additively to inhibit matrix gene expression by chondrocytes. Arthritis Res Theory 2005, 7:R127-R138.

31. Usmani SE, Appleton CT, Beier F: Transforming growth factor-alpha induces endothelin receptor-A expression in osteoarthritis. J Orthp Res 2012, 30:1391-1397.

32. Appleton CT, Usmani SE, Bernier SM, Aigner T, Beier F: Transforming growth factor alpha suppression of articular chondrocyte phenotype and Sox9 expression in a rat model of osteoarthritis. Arthritis Rheum 2007, 56:3693-3705.

33. Jakob M, Demarteau O, Schafer D, Hintermann B, Dick W, Heberer M, Martin I: Specific growth factors during the expansion and redifferentiation of adult human articular chondrocytes enhance chondrogenesis and cartilaginous tissue formation in vitro. J Cell Biochem 2001, 81:368-377.

34. Huh Y-H, Kim S-H, Kim S-J, Chun J-S: Differentiation status-dependent regulation of cyclooxygenase-2 expression and prostaglandin E2 production by epidermal growth factor via mitogen-activated protein kinase in articular chondrocytes. J Biol Chem 2003, 278:9691-9697.

35. Takeda H, Inoue H, Kutsuna T, Matsushita N, Takahashi T, Watanabe S, Higashiyama S, Yamamoto $\mathrm{H}$ : Activation of epidermal growth factor receptor gene is involved in transforming growth factor- $\beta$-mediated fibronectin expression in a chondrogenic progenitor cell line. Int J Mol Med 2010, 25:593-600.

36. Appleton CT, McErlain DD, Henry JL, Holdsworth DW, Beier F: Molecular and histological analysis of a new rat model of experimental knee osteoarthritis. Ann NY Acad Sci 2007, 1117:165-174.

37. Hackel PO, Gishizky M, Ullrich A: Mig6 is a negative regulator of the Epidermal Growth Factor Receptor signal. Biol Chem 2001, 382:1649-1662.

38. Logan M, Martin JF, Nagy A, Lobe C, Olson EN, Tabin CJ: Expression of Cre Recombinase in the developing mouse limb bud driven by a Prx1 enhancer. Genesis 2002, 33:77-80.

39. Jin N, Gilbert JL, Broaddus RR, DeMayo FJ, Jeong J-W: Generation of a Mig6-conditional null allele. Genesis 2007, 45:716-721.

40. Fisher M, Meyer C, Garber G, Dealy CN: Role of IGFBP2, IGF-I and IGF-II in regulating long bone growth. Bone 2005, 37:741-750.

41. Watkins DJ, Zhou Y, Chen CL, Darbyshire A, Besner GE: Heparan-binding epidermal growth factor like-growth factor protects mesenchymal stem cells. J Surg Res 2012, 177:359-364.

42. Kerpedjieva SS, Kim DS, Barbeau DJ, Tamama K: EGFR ligands drive multipotential stromal cells to produce multiple growth factors and cytokines via early growth response-1. Stem Cells 2012, 21:2541-2551.

43. Omi M, Maihle NJ, Fisher M, Dealy CN: Studies on EGF receptor signaling in vertebrate limb patterning. Dev Dyn 2005, 233:288-300.

44. Fisher MC, Clinton GM, Maihle NJ, Dealy CN: Requirement for ErbB2/ErbB signaling in developing cartilage and bone. Dev Growth Differ 2007, 49:503-513.

45. Dexheimer V, Frank S, Richter W: Proliferation as a requirement for in vitro chondrogenesis of human mesenchymal stem cells. Stem Cells Dev 2012, 21:2160-2169.

46. Karlsson C, Thornemo M, Henricksson HB, Lindahl A: Identification of a stem cell niche in the zone of Ranvier. J Anat 2009, 215:355-363.

47. Dy $P$, Smits $P$, Silvester A, Penzo-Méndez A, Dumitriu B, Han Y, de la Motte CA, Kingsley DM, Lefebvre $V$ : Synovial joint morphogenesis requires the chondrogenic action of Sox 5 and Sox6 in growth plate and articular cartilage. Dev Biol 2010, 341:346-359.

48. Koyama E, Shibukawa Y, Nagayama M, Sugito H, Young B, Yuasa T, Okabe T, Ochiai T, Kamiya N, Rountree RB, Kingsley DM, Iwamoto M, EnomotoIwamoto M, Pacifici M: A distinct cohort of progenitor cells participates in synovial joint and articular cartilage formation during mouse limb skeletogenesis. Dev Biol 2008, 316:62-73.

49. van der Kraan PM, Blaney Davidson EN, Van den Berg WB: A role for agerelated changes in TGF $\beta$ signaling in aberrant chondrocyte differentiation and osteoarthritis. Arthritis Res Ther 2010, 12:201.

50. Lee SY, Nakagawa T, Reddi AH: Induction of chondrogenesis and superficial zone protein (SZP)/lubricin expression by mesenchymal progenitors in the infrapatellar fat pad of the knee joint treated with TGF- $\beta 1$ and BMP-7. Biochem Biophy Res Comm 2008, 376:148-153.

51. Zhu M, Tang D, Wu Q, Hao S, Chen M, Xie C, Rosier RN, O'Keefe RJ, Zuscik M, Chen D: Activation of $\beta$-catenin signaling in articular 
chondrocytes leads to an osteoarthritic like phenotype in adult beta catenin conditional activation mice. J Bone Miner Res 2009, 24:12-21.

52. Yuasa T, Kondo N, Yasuhara R, Shimono K, Mackem S, Pacifici M, Iwamoto M, Enomoto-Iwamoto M: Transient activation of Wnt/betacatenin signaling induces abnormal growth plate closure and articular cartilage thickening in postnatal mice. Am J Pathol 2009, 175:1933-2003.

53. Uchiyama-Tanaka Y, Matsubara H, Mori Y, Kosaki A, Kishimoto N, Amano K, Higashiyama S, Iwasaka T: Involvement of the HB-EGF and EGF receptor transactivation in TGF-beta mediated fibronectin expression in mesangial cells. Kidney Int 2002, 62:799-808.

54. Wendt MK, Smith JA, Schiemann WP: Transforming growth factor- $\beta$ induced epithelial-mesenchymal transition facilitates epidermal growth factor dependent breast cancer progression. Oncogene 2010, 29:6485-6498

55. HU T, Li C: Convergence between Wnt- $\beta$-catenin and EGFR signaling in cancer. Mol Cancer 2010, 9:236.

56. Yue $X$, Lan F, Yang W, Yang Y, Han L, Zhang A, Liu J, Zeng H, Jiang T, Pu P, Kang C: Interruption of $\beta$-catenin suppresses the EGFR pathway by blocking multiple oncogenic targets in human glioma cells. Brain Res 2010, 1366:27-37.

57. Guteri KK, Mandal T, Chatterjee A, Sarkar M, Bhattacharya S, Chatterjee U, Ghosh MK: Mechanism of $\beta$-catenin mediated transcriptional regulation of epidermal growth factor receptor expression in glycogen synthase kinase 2 B-inactivated prostate cancer cells. J Biol Chem 2012, 287:18287-18296.

58. Segatto O, Anastasi S, Alema S: Regulation of epidermal growth factor receptor signaling by inducible feedback inhibitors. J Cell Sci 2011, 124:1785-1793.

59. Zhang Y-W, Vande Woude GF: Mig-6, signal transduction, stress response and cancer. Cell Cycle 2007, 6:507-513.

60. Wilken JA, Badri T, Cross S, Raji R, Santin AD, Schwartz P, Branscum AJ, Baron AT, Sakhitab Al, Maihle NJ: EGFR/HER-targeted therapeutics in ovarian cancer. Future Med Chem 2012, 4:447-469.

61. Ferby I, Reschke M, Kudlacek O, Knyazev P, Pante G, Amann K, Sommergruber W, Kraut N, Ullrich A, Fassler R, Kelin R: Mig6 is a negative regulator of EGF receptor mediated skin morphogenesis and tumor formation. Nat Med 2006, 12:568-573.

62. Burton-Wurster N, Mateescu RG, Todhunter RJ, Clements KM, Sun Q Scarpino V, Lust G: Genes in canine articular cartilage that resond to mechanical injury: gene expression studies with Affymetrix canine GeneChip. J Hered 2005, 96:821-828.

63. Gomez-Camarillo MA, Almonte-Becerril M, Vasquez Tort M, Tapia-Ramirez J, Kouri Flores JB: Chondrocyte proliferation in a new culture system. Cell Prolif 2009, 442:207-218.

64. Beerlie RR, Hynes NE: Epidermal growth factor related peptides activate distinct subsets of ErbB receptors and differ in their biological activities. J Biol Chem 1996, 271:6071-6076.

65. Olayioye MA, Neve RM, Lane HA, Hynes NE: The ErbB signaling network: receptor heterodimerization in development and cancer. EMBO J 2000, 19:3159-3167.

66. Fiorentino L, Pertica C, Fiorini M, Talora C, Crescenzi M, Castellani L, Alema S, Benedetti P, Segatto O: Inhibition of ErbB2-mitogenic and transforming activity by RALT, a mitogen-induced signal transducer which binds to the ErbB-2 kinase domain. Mol Cell Biol 2000, 20:7735-7750.

67. Lin C-L, Du J, Shen WT, Whang EE, Donner DB, Griff N, He F, Moore FD, Clark OH, Ruan DT: Mitogen-inducible gene- 6 is a multifunctional adapter protein with tumor suppressor like activity in papillary thyroid cancer. J Clin Endocrinol Metab 2011, 96:E554-E565.

68. Pante G, Thompson J, Lamballe F, Iwata T, Ferby I, Barr FA, Davies AM, Maina F, Klein R: Mitogen-inducible gene- 6 is an endogenous inhibitor of HGF/Met-induced cell migration and neurite outgrowth. J Cell Biol 2005, 171:337-348.

69. Bau B, McKenna LA, Soeder S, Fan Z, Pecht A, Aigner T: Hepatocyte growth factor/scatter factor is not a potent regulator of anabolic and catabolic gene expression in adult normal articular chondrocytes. Biochem Biophys Res Commun 2004, 316:984-990.

70. Hughes LC, Archer CW, ap Gwynn I: The ultrastructure of mouse articular cartilage: collagen orientation and implications for tissue functionality. A polarized light and scanning electron microscope study and review. Eur Cell Mater 2005, 9:68-84.
71. Goldring MB: Chondrogenesis, chondrocyte differentiation, and articular cartilage metabolism in health and osteoarthritis. Ther Adv Musculoskelet Dis 2012, 4:269-285.

72. van der Kraan PM, Van den Berg WB: Chondrocyte hypertrophy and osteoarthritis: role in initiation and progression of cartilage degeneration? Osteoarthritis Cart 2012, 20:223-232.

\section{doi:10.1186/ar4233}

Cite this article as: Shepard et al:: Transient anabolic effects accompany epidermal growth factor receptor signal activation in articular cartilage in vivo. Arthritis Research \& Therapy 2013 15:R60.

\section{Submit your next manuscript to BioMed Central and take full advantage of:}

- Convenient online submission

- Thorough peer review

- No space constraints or color figure charges

- Immediate publication on acceptance

- Inclusion in PubMed, CAS, Scopus and Google Scholar

- Research which is freely available for redistribution

Submit your manuscript at www.biomedcentral.com/submit
C Biomed Central 